\title{
SUR LA SYNTHÈSE DU SUCRE DU LAIT
}

Par A. PICTET et H. VOGEL.

On sait depuis fort longtemps (Dubrunfaut, 1856) que le sucre de lait est assez facilement hydrolysé par les acides, en se convertissant en un mélange de deux sucres plus simples, le glucose et le galactose. L'opération inverse, qui consisterait à régénérer le lactose par l'union de ses deux constituants, a été souvent tentée, mais sans succès. La cause de ees échees est aujourd'hui connue; elle réside dans le fait que le glucose et le galactose peuvent exister sous deux formes distinctes, que l'on a désignées par les lettres grecques a et $\beta$. Or ce sont exclusivement les formes $\alpha$ que l'on retire pratiquement du lactose, tandis qu'il a été prouvé, d'une part, que c'est le galactose $\beta$ qui entre dans la composition de ce disaccharide, et d'autre part que le glucose $\beta$ en raison de sa stabilité vis-à-vis de la chæleur, peut seul se prêter à une condensation.

Le problème ne semblait done pouvoir être résolu qu'en répétant les essais avec les deux modifications $\beta$ comme points de départ. Elles sont toutes deux connues, ayant été préparées en 1896 par C. Tanret en faisant cristalliser dans certaines conditions très précises les sucres. Nous avons réussi à les combiner l'une à l'autre en mettant à profit une des propriétés des anhydrides des sucres, constatée par l'un de nous et ses collaborateurs. Il a été reconnu, en effet, que la plupart des sucres réducteurs perdent une molécule d'eau lorsqu'on les chauffe à 140-150 dans le vide, et se transforment en anhydrides internes. C'estainsi que le glucose $\mathrm{C}^{6} \mathrm{H}^{12} \mathrm{O}^{6}$ se convertit en glucosane $\mathrm{C}^{6} \mathrm{H}^{10} \mathrm{O}^{5}$, le fructose en fructosane, le maltose $\mathrm{C}^{12} \mathrm{H}^{22} \mathrm{O}^{11}$ en maltosane $\mathrm{C}^{12} \mathrm{H}^{20} \mathrm{O}^{10}$, etc. Or ces corps possèdent la propriété commune de former des produits d'addition avec divers autres composés, et en particulier avec ceux qui possèdent des oxhydriles alcooliques (alcools, glycol, glycérine, sucres). C'est en nous basant sur ce fait que nous avons pu, il y a quelques mois, réaliser une synthèse du maltose, en chauffant à $170^{\circ}$ un mélange équimoléculaire de glucosane et de $\beta$-glucose. La réaction s'est effectuée selon l'équation suivante :

\section{$\mathrm{C}^{5} \mathrm{H}^{12} \mathrm{O}^{5}+\mathrm{C}^{5} \mathrm{H}^{10} \mathrm{O}^{6}=\mathrm{C}^{12} \mathrm{H}^{22} \mathrm{O}^{11}$ \\ $\beta$-Glucose Glueosane Maltose}

II nous a semblé que ce procédé si simple pourrait se prêter à la synthèse d'autres disaccharides, et en particul.er du lactose. Il devait suffire, dans ce dernier cas, de remplacer, dans l'expérience précédente, l'anhydride du glucose par celui du galactose $\beta$.

Malheureusement, ce dernier anhydride n'était pas connu. II nous a donc fallu commencer par le préparer. Nous y sommes arrivés facilement par le procédé qui nous avait foumi les autres anhydrides, c'est 
à-dire en chauffant à $150^{\circ}$ dans le vide le galactose $\beta$. La $\beta$-galactosane ainsi obtenue est un corps cristallin, fusible à $154-155^{\circ}$, très hygroscopique et possédant un pouvoir rotatoire de $+30^{\circ} 5$. Nous l'avons mélangée à un poids égal de $\beta$-glucose et à quelques centigrammes de chlorure de zinc, et nous avons chauffé le tout pendant une demiheure à $150^{\circ}$ dans le vide. La masse entre en fusion, puis se resolidifie pou à peu ; on n'observe aucun dégagement d'eau ; il y a simple addition des deux molésules, selon l'équation:

$$
\begin{aligned}
& \mathrm{C}^{6} \mathrm{H}^{12} \mathrm{O}^{6}+\mathrm{C}^{6} \mathrm{H}^{10} \mathrm{O}^{5}=\mathrm{C}^{12} \mathrm{H}^{22} \mathrm{O}^{11} \\
& \beta \text {-Glucose } \beta \text {-Galactosane Lactose }
\end{aligned}
$$

Après refroidissement dans le vide, le produit de cette réaction est dissous dans l'eau; on fait bouillir cette solution, d'abord avec un peu de carbonate d'argent pour précipiter à la fois le chlore et le zine, puis avec du charbon animal, et on l'évapore à sec. Le résidu est traité par l'anhydride acétique; on obtient ainsi un acétate facile à purifier par cristallisation et fusible à $85^{\circ}$. Ce point de fusion est celui du $\beta$-octacétate de lactose.

Nous avons pu obtenir ce même acétate d'une manière plus simple encore, 'en chauffant le $\beta$-glucose, non plus avec la $\beta$-galactosane, mais avec le $\beta$-galactose. Le résultat est le même; il faut seulement élever la température à $175^{\circ}$. Dans ces conditions le glucose $\beta$ reste inaltéré, le $\beta$-galactose seul est transformé en son anhydride. On se retrouve donc danś les mêmes conditions que précédemment.

L'axétate obtenu par ces deux procédés a été ensuite saponifié par l'éthylate de sodium, suivant la méthode de Zemplen. Cette opération nous a fourni un sucre réducteur possédant la formule $\mathrm{C}^{12} \mathrm{H}^{22} \mathrm{O}^{11}$. $\mathrm{H}^{2} \mathrm{O}$ et les propriétés suivantes :

Petits cristaux incolores, fusibles à $201^{\circ}$.

Saveur à peine sucrée.

Soluble dans 6 parties d'eau froide et dans 2,8 parties d'eau bouillante Pouvoir rotatoire $+80^{\circ}, 7$, s'abaissant en 24 heures à $+51^{\circ}, 8$.

Poids moléculaire, déterminé par cryoscopie dans l'eau : 359.

Point de fusion de l'osazone : $200^{\circ}$.

$" \Rightarrow$ de l'octonitrate : $144^{\circ}$.
$"$ du $\beta$-octacétate : $85^{\circ}$.
Pouvoir rotatoire du $\beta$-octacétate : $-4^{\circ}, 5$.

Si l'on compare ces chiffres à ceux qui caractérisent le sucre de lait, on constate entre eux la plus parfaite concordance ; l'identité des deux substances se trouve ainsi établie.

Genève, Laboratoire de Chimie organique de l'Université. 